\title{
IC 751: A New Changing Look AGN Discovered By NuSTAR
}

Ricci, C.; Bauer, F. E.; Arevalo, P.; Boggs, S.; Brandt, W. N.; Christensen, Finn Erland; Craig, W. W.; Gandhi, P.; Hailey, C. J.; Harrison, F. A.

Total number of authors:

15

Published in:

Astrophysical Journal

Link to article, DOI:

$10.3847 / 0004-637 X / 820 / 1 / 5$

Publication date:

2016

Document Version

Publisher's PDF, also known as Version of record

Link back to DTU Orbit

Citation (APA):

Ricci, C., Bauer, F. E., Arevalo, P., Boggs, S., Brandt, W. N., Christensen, F. E., Craig, W. W., Gandhi, P. Hailey, C. J., Harrison, F. A., Koss, M., Markwardt, C. B., Stern, D., Treister, E., \& Zhang, W. W. (2016). IC 751: A New Changing Look AGN Discovered By NuSTAR. Astrophysical Journal, 820(1), [5]. https://doi.org/10.3847/0004-637X/820/1/5

\section{General rights}

Copyright and moral rights for the publications made accessible in the public portal are retained by the authors and/or other copyright owners and it is a condition of accessing publications that users recognise and abide by the legal requirements associated with these rights.

- Users may download and print one copy of any publication from the public portal for the purpose of private study or research.

- You may not further distribute the material or use it for any profit-making activity or commercial gain

- You may freely distribute the URL identifying the publication in the public portal 


\title{
IC 751: A NEW CHANGING LOOK AGN DISCOVERED BY NUSTAR
}

\author{
C. Ricci ${ }^{1,2}$, F. E. Bauer ${ }^{1,2,3,4}$, P. Arevalo ${ }^{5}$, S. Boggs $^{6}$, W. N. Brandt ${ }^{7,8,9}$, F. E. Christensen ${ }^{10}$, W. W. Craig ${ }^{6}$, P. Gandhi ${ }^{11}$, \\ C. J. Hailey ${ }^{12}$, F. A. Harrison ${ }^{13}$, M. Koss ${ }^{14}$, C. B. Markwardt ${ }^{15,16}$, D. Stern ${ }^{17}$, E. Treister ${ }^{2,18}$, And W. W. Zhang ${ }^{16}$ \\ ${ }^{1}$ Instituto de Astrofísica, Facultad de Física, Pontificia Universidad Católica de Chile, Casilla 306, Santiago 22, Chile; cricci@astro.puc.cl \\ ${ }^{2}$ EMBIGGEN Anillo, Concepción, Chile \\ ${ }^{3}$ Millennium Institute of Astrophysics, Vicuña Mackenna 4860, 7820436 Macul, Santiago, Chile \\ ${ }^{4}$ Space Science Institute, 4750 Walnut Street, Suite 205, Boulder, CO 80301, USA \\ ${ }^{5}$ Instituto de Física y Astronomía, Facultad de Ciencias, Universidad de Valparaíso, Gran Bretana N 1111, Playa Ancha, Valparaíso, Chile. \\ 6 Space Sciences Laboratory, University of California, Berkeley, CA 94720, USA \\ ${ }^{7}$ Department of Astronomy and Astrophysics, The Pennsylvania State University, University Park, PA 16802, USA \\ ${ }_{9}^{8}$ Institute for Gravitation and the Cosmos, The Pennsylvania State University, University Park, PA 16802, USA \\ ${ }^{9}$ Department of Physics, 104 Davey Lab, The Pennsylvania State University, University Park, PA 16802, USA \\ ${ }^{10}$ DTU Space, National Space Institute, Technical University of Denmark, Elektronvej 327, DK-2800 Lyngby, Denmark \\ ${ }^{11}$ School of Physics \& Astronomy, University of Southampton, Highfield, Southampton SO17 1BJ, UK \\ ${ }^{12}$ Columbia Astrophysics Laboratory, Columbia University, New York 10027, USA \\ ${ }^{13}$ Cahill Center for Astronomy and Astrophysics, California Institute of Technology, Pasadena, CA 91125, USA \\ ${ }^{14}$ Institute for Astronomy, Department of Physics, ETH Zurich, Wolfgang-Pauli-Strasse 27, CH-8093 Zurich, Switzerland \\ ${ }^{15}$ Department of Astronomy, University of Maryland, College Park, MD 20742, USA \\ ${ }^{16}$ Astroparticle Physics Laboratory, Mail Code 661, NASA Goddard Space Flight Center, Greenbelt, MD 20771, USA \\ ${ }^{17}$ Jet Propulsion Laboratory, California Institute of Technology, Pasadena, CA 91109, USA \\ ${ }^{18}$ Universidad de Concepción, Departamento de Astronomía, Casilla 160-C, Concepción, Chile \\ Received 2015 November 28; accepted 2016 January 20; published 2016 March 10
}

\begin{abstract}
We present results of five Nuclear Spectroscopic Telescope Array (NuSTAR) observations of the type 2 active galactic nucleus (AGN) in IC 751, three of which were performed simultaneously with XMM-Newton or Swift/ $\mathrm{X}$-Ray Telescope. We find that the nuclear X-ray source underwent a clear transition from a Compton-thick $\left(N_{\mathrm{H}} \simeq 2 \times 10^{24} \mathrm{~cm}^{-2}\right)$ to a Compton-thin $\left(N_{\mathrm{H}} \simeq 4 \times 10^{23} \mathrm{~cm}^{-2}\right)$ state on timescales of $\lesssim 3$ months, which makes IC 751 the first changing look AGN discovered by NUSTAR. Changes of the line of sight column density at the $\sim 2 \sigma$ level are also found on a timescale of $\sim 48 \mathrm{hr}\left(\Delta N_{\mathrm{H}} \sim 10^{23} \mathrm{~cm}^{-2}\right)$. From the lack of spectral variability on timescales of $\sim 100 \mathrm{ks}$, we infer that the varying absorber is located beyond the emission-weighted average radius of the broad-line region (BLR), and could therefore be related either to the external part of the BLR or a clumpy molecular torus. By adopting a physical torus X-ray spectral model, we are able to disentangle the column density of the non-varying absorber $\left(N_{\mathrm{H}} \sim 3.8 \times 10^{23} \mathrm{~cm}^{-2}\right)$ from that of the varying clouds $\left[N_{\mathrm{H}} \sim(1-150)\right.$ $\left.\times 10^{22} \mathrm{~cm}^{-2}\right]$, and to constrain that of the material responsible for the reprocessed X-ray radiation $\left(N_{\mathrm{H}} \sim 6 \times 10^{24} \mathrm{~cm}^{-2}\right)$. We find evidence of significant intrinsic X-ray variability, with the flux varying by a factor of five on timescales of a few months in the 2-10 and 10-50 keV band.
\end{abstract}

Key words: galaxies: active - galaxies: individual: (IC 751) - galaxies: Seyfert - X-rays: galaxies

\section{INTRODUCTION}

Variability of the line of sight column density $\left(N_{\mathrm{H}}\right)$ might be rather common in active galactic nuclei (AGNs; Risaliti et al. 2002; Bianchi et al. 2012; Torricelli-Ciamponi et al. 2014), and in the past decade several objects have been found to show eclipses of the X-ray source, both due to Compton-thick (CT; $N_{\mathrm{H}} \gtrsim 10^{24} \mathrm{~cm}^{-2}$ ) and to Compton-thin $\left(N_{\mathrm{H}}<10^{24} \mathrm{~cm}^{-2}\right)$ material. Since the X-ray source is believed to be located very close to the supermassive black hole (SMBH), this variable absorption could be associated either with broad-line region (BLR) clouds, or with clumps in the molecular torus. In at least a few cases (e.g., Risaliti et al. 2009; Maiolino et al. 2010) these variations have been found to occur on timescales of days, and are consistent with being related to material in the BLR. Markowitz et al. (2014) have recently shown, by studying RXTE light curves of 55 AGNs, that for eight objects of their sample there seems to be variation of absorbing material also on longer timescales (months to years). They associated these changes in $N_{\mathrm{H}}$ with clumps in the molecular torus. Interestingly, none of the eclipses detected by Markowitz et al. (2014) were due to CT material.
So far variations in the $N_{\mathrm{H}}$ of the neutral absorber have been found in more than 20 AGNs including 1H 0419-577 (Pounds et al. 2004), Centaurus A (Beckmann et al. 2011; Rivers et al. 2011), ESO 323-G77 (Miniutti et al. 2014), H0557-385 (Longinotti et al. 2009), MR 2251-178, Mrk 348, Mrk 509 (Markowitz et al. 2014), Mrk 6 (Immler et al. 2003), Mrk 766 (Risaliti et al. 2011), Mrk 79 (Markowitz et al. 2014), NGC 1068 (Marinucci et al. 2016), NGC 1365 (Risaliti et al. 2005, 2007; Maiolino et al. 2010; Walton et al. 2014; Rivers et al. 2015b), NGC 3227 (Lamer et al. 2003), NGC 3783 (Markowitz et al. 2014), NGC 4151 (Puccetti et al. 2007), NGC 4388 (Elvis et al. 2004), NGC 4395 (Nardini \& Risaliti 2011), NGC 4507 (Braito et al. 2013; Marinucci et al. 2013), NGC 454 (Marchese et al. 2012), NGC 5506 (Markowitz et al. 2014), NGC 6300 (Guainazzi 2002), NGC 7582 (Piconcelli et al. 2007; Bianchi et al. 2009; Rivers et al. 2015a), NGC 7674 (Bianchi et al. 2005), PG 2112+059 (Gallagher et al. 2004), UGC 4203 (Guainazzi et al. 2002; Risaliti et al. 2010), and SWIFT J2127.4+5654 (Sanfrutos et al. 2013). In most cases these variations are due to Comptonthin material, and only for a handful of sources is the varying absorber CT (i.e., ESO 323-G77, NGC 1068, NGC 1365, 
Table 1

X-Ray Observations Log

\begin{tabular}{|c|c|c|c|c|}
\hline Obs. \# & Facility & Observation Date & Observation ID & Net Exposure (ks) \\
\hline 1 & Swift $/ \mathrm{XRT}^{\mathrm{a}}$ & 2008 Feb 20 13:13:01 & 00037374001 & 2.3 \\
\hline 2 & NUSTAR & 2012 Oct 28 23:01:07 & 60061217002 & 13.1 \\
\hline 3 & NUSTAR & 2013 Feb 04 00:26:07 & 60061217004 & 52.0 \\
\hline 4 & NUSTAR & 2013 May 23 05:36:07 & 60061217006 & 25.0 \\
\hline 4 & Swift/XRT & 2013 May 25 16:38:59 & 00080064001 & 5.8 \\
\hline 5 & NUSTAR & 2014 Nov 28 06:01:07 & 60001148002 & 26.3 \\
\hline 5 & XMM-Newton & 2014 Nov 28 13:20:42 & 0744040301 & $18.4^{\mathrm{b}} ; 23.1^{\mathrm{c}}$ \\
\hline 6 & NUSTAR & 2014 Nov 30 06:26:07 & 60001148004 & 25.7 \\
\hline 6 & XMM-Newton & 2014 Nov 30 13:11:46 & 0744040401 & $18.2^{\mathrm{b}} ; 22.4^{\mathrm{c}}$ \\
\hline
\end{tabular}

Notes.

a This observation was not used for spectral fitting because of the low number of counts.

b $\mathrm{EPIC} / \mathrm{PN}$.

${ }^{\mathrm{c}}$ EPIC MOS1 and MOS2.

NGC 454, NGC 6300, NGC 7582, NGC 7674, UGC 4203). AGNs switching between Compton-thin and CT states are usually called changing look AGNs (e.g., Matt et al. 2003) because their spectral shape changes dramatically (from transmission-dominated to reflection-dominated). In the optical band, changing look AGNs are objects that transition from type 1 to type 1.8/1.9/2 (e.g., LaMassa et al. 2015), or from type $1.8 / 1.9 / 2$ to type 1 (e.g., Shappee et al. 2014). In the following, we will refer to the X-ray classification only.

IC $751(z=0.0311, D=137 \mathrm{Mpc}$; Falco et al. 1999) is a type 2 AGN (Véron-Cetty \& Véron 2010) in an edge-on spiral galaxy (de Vaucouleurs et al. 1991) that has not yet been studied in detail in the X-ray band. The source was reported in the 70 month Swift/Burst Alert Telescope (BAT) catalog (Baumgartner et al. 2013), and was observed by Nuclear Spectroscopic Telescope Array (NUSTAR) as part of the campaign aimed at following up on Swift/BAT-detected sources (M. Balokovic et al. 2016, in preparation). The interesting X-ray characteristics of this object triggered several follow-up observations with NUSTAR. We report here on the five NUSTAR observations of this source carried out between 2012 and 2014, two of which were performed jointly with XMM-Newton. The source switches from a CT to Comptonthin state between the different observations, and is the first changing look AGN discovered by NuSTAR. Following our $\mathrm{X}$-ray spectral and temporal analysis, we put constraints on the location of the varying obscuring material. Throughout the paper, we consider a luminosity distance of the source of $d_{\mathrm{L}}=135 \mathrm{Mpc}$, and adopt standard cosmological parameters $\left(H_{0}=70 \mathrm{~km} \mathrm{~s}^{-1} \mathrm{Mpc}^{-1}, \Omega_{\mathrm{m}}=0.3, \Omega_{\Lambda}=0.7\right)$. Unless otherwise stated, all uncertainties are quoted at the $90 \%$ confidence level.

\section{X-RAY OBSERVATIONS AND DATA REDUCTION}

IC 751 was observed five times by NUSTAR, twice jointly with XMM-Newton (PI F. Bauer), and two times by the Swift/ $\mathrm{X}$-Ray Telescope (XRT). Details about these observations are reported in Table 1.

\subsection{NUSTAR}

$N u S T A R$ (Harrison et al. 2013) is the first focusing X-ray telescope in orbit operating above $10 \mathrm{keV}$. NuSTAR consists of two focal-plane modules (FPMA and FPMB), both operating in the 3-79 keV band and with similar characteristics.
NuSTAR observed IC 751 five times between 2012 October and 2014 November, with exposure times ranging between 13 and $52 \mathrm{ks}$ (Table 1). The data collected by NuSTAR were processed using the NUSTAR Data Analysis Software NUSTARDAS v1.4.1 within Heasoft v6.16, using the latest calibration files, released in 2015 March (Madsen et al. 2015). The source spectra and light curves were extracted using the NUPRODUCTS task, selecting circular regions with a radius of $50^{\prime \prime}$. The background spectra and light curves were obtained in a similar fashion, using a circular region of $60^{\prime \prime}$ radius located where no other source was detected. The source light curve was corrected for background using the LCMATH task.

\subsection{XMM-Newton}

The XMM-Newton X-ray observatory (Jansen et al. 2001) observed IC 751 twice at the end of 2014 November. We analyzed the two $20 \mathrm{ks}$ XMM-Newton observations of IC 751, taking into account the data obtained by the PN (Strüder et al. 2001) and MOS (Turner et al. 2001) cameras. The observation data files (ODFs) were reduced using the the XMM-Newton Standard Analysis Software version 12.0.1 (Gabriel et al. 2004). The raw PN (MOS) data files were then processed using the EPCHAIN (EMCHAIN) task.

For both observations we analyzed the background light curves in the 10-12 keV band (EPIC/PN), and above $10 \mathrm{keV}$ (EPIC/MOS), to filter the exposures for periods of high background activity. We set the threshold to $0.5 \mathrm{ct} \mathrm{s}^{-1}$ and to $0.3 \mathrm{ct} \mathrm{s}^{-1}$ for PN and MOS, respectively. This resulted in about $10 \%$ of the observations being filtered out. We report the final exposures used in Table 1. Only patterns that correspond to single and double events (PATTERN $\leqslant 4$ ) were selected for $\mathrm{PN}$, and corresponding to single, double, triple, and quadruple events for MOS (PATTERN $\leqslant 12$ ).

For the three cameras, the source spectra were extracted from the final filtered event list using circular regions centered on the object, with a radius of $20^{\prime \prime}$, while the background was estimated using circular regions with a radius of $40^{\prime \prime}$ located on the same CCD as the source, where no other source was present. No pile up was detected for any of the three cameras in the two observations. The ARFs and RMFs were created using the ARFGEN and RMFGEN tasks, respectively. For both observations the source and background spectra of the two MOS cameras, together with the RMF and ARF files, were merged using the ADDASCASPEC task. 


\subsection{Swift}

IC 751 was observed twice by the XRT (Burrows et al. 2005) on board the Swift observatory (Gehrels et al. 2004): for $2.3 \mathrm{ks}$ in 2008 February and for $5.8 \mathrm{ks}$, two days after the NUSTAR observation, in 2013 May. During the first observation only five counts were detected, so that no detailed spectral analysis could be performed. The data were reduced using the XRTPIPELINE V0.13.0, which is part of the XRT Data Analysis Software within Heasoft v6.16.

The BAT on board Swift has been monitoring the sky in the 14-195 keV band since 2005, and has so far detected more than 800 AGNs (Baumgartner et al. 2013), 55 of which are CT sources (Ricci et al. 2015). Given the significant $N_{\mathrm{H}}$ variability of IC 751 found by NuSTAR, we did not use the 70 month stacked Swift/BAT spectrum for our spectral analysis. The long-term variability inferred by Swift/BAT will be discussed in Section 5. The 70 month averaged flux of IC 751 in the $14-195 \mathrm{keV}$ band is $13.1_{-3.6}^{+3.9} \times 10^{-12} \mathrm{erg} \mathrm{s}^{-1} \mathrm{~cm}^{-2} \quad(90 \%$ confidence interval).

\section{X-RAY SPECTRAL ANALYSIS-SLAB MODEL}

We performed X-ray spectral analysis with XSPEC v.12.8.2 (Arnaud 1996). To all models we added a photoelectric absorption component (TBABS; Wilms et al. 2000) to take into account Galactic absorption in the direction of the source, fixing the value of the column density to $N_{\mathrm{H}}^{\mathrm{G}}=$ $1.2 \times 10^{20} \mathrm{~cm}^{-2}$ (Kalberla et al. 2005). In order to use $\chi^{2}$ statistics, NuSTAR FPMA/FPMB and XMM-Newton EPIC PN and MOS spectra were binned to have at least 20 counts per bin. Quoted errors correspond to a $90 \%$ confidence level $\left(\Delta \chi^{2}=2.7\right)$. Given the low signal-to-noise of the Swift/XRT spectrum of Observation 4, we did not bin the spectrum, and applied Cash statistics (Cash 1979; CSTAT in XSPEC).

As a first step to shed light on the spectral variability, we used a model which considers reprocessed radiation from a slab to reproduce the X-ray spectrum of IC 751. Since the first two data sets lacked simultaneous coverage below $5 \mathrm{keV}$, and the Swift/XRT observation in Observation 4 did not have a very high signal-to-noise ratio, we used the two joint NUSTAR/ $X M M$-Newton spectra (Observations 5 and 6, Section 3.1) to constrain the fundamental parameters (photon index, normalization of the reflection component, normalization of the $\mathrm{Fe} \mathrm{K} \alpha$ line), and then used this information to fit the other observations (Observations 2, 3, and 4, Section 3.2), in order to constrain the value of the line of sight column density, $N_{\mathrm{H}}$, and the normalization of the X-ray primary emission. Observation 1 was not used due to its poor statistics. It was, however, possible to infer the $2-10 \mathrm{keV}$ flux of the X-ray source during this observation $\left(3.1_{-2.4}^{+0.4} \times 10^{-13} \mathrm{erg} \mathrm{s} \mathrm{cm}^{-2}\right)$, which is consistent with that of Observation 3.

\subsection{Observations 5 and 6}

The slab model we used to analyze the broadband X-ray spectrum of IC 751 includes: (i) a power law with a highenergy cutoff, which represents the primary X-ray emission; (ii) photoelectric absorption and Compton scattering, to take into account the line of sight obscuration; (iii) unabsorbed X-ray reprocessed radiation; (iv) a Gaussian line to reproduce the $\mathrm{Fe} \mathrm{K} \alpha$ emission; (v) a cutoff power-law component to reproduce the scattered X-ray emission; (vi) a thermal plasma model, to take into account a possible contribution of the host galaxy to the X-ray spectrum below $\sim 2 \mathrm{keV}$. It must be stressed that the emission below $2 \mathrm{keV}$, which cannot be accounted for by the scattered component alone, could also be due to the blending of emission lines created by photo-ionization. However, due to the limited energy resolution of our data, in the following we will use a thermal plasma model. To reproduce photoelectric absorption and Compton scattering, we used the TBABS and CABS models, respectively. We took into account the reprocessed X-ray radiation using the PEXRAV model (Magdziarz \& Zdziarski 1995), which assumes reflection from a semi-infinite slab. The value of the reflection parameter $(R)$ was set to be negative in order to include only the reprocessed component. The values of the photon index $(\Gamma)$, cutoff energy $\left(E_{\mathrm{C}}\right)$, and the normalization were fixed to the values of the cutoff power law, while the inclination angle of the observer with respect to the reflecting slab was set to $i=30^{\circ}$. This angle was selected considering the type 2 nature of IC 751, and assuming that the reflecting material is associated with the molecular torus. The width of the $\mathrm{Fe} \mathrm{K} \alpha$ line $(\sigma)$ was fixed to $40 \mathrm{eV}$, a value well below the energy resolution of XMM-Newton and NUSTAR, while the energy and normalization of the line were left free to vary. The width of the $\mathrm{Fe} \mathrm{K} \alpha$ was chosen considering that the bulk of the line is created in material located in the BLR or in the molecular torus (e.g., Shu et al. 2010; Ricci et al. 2014a, 2014b). The scattered $\mathrm{X}$-ray emission was taken into account by multiplying an additional unabsorbed cut off power law by a constant $\left(f_{\text {scatt }}\right.$, typically of the order of a few percent), fixing all the parameters to those of the primary X-ray emission. A multiplicative constant to include possible cross-calibration offset between the different instruments was added, and was found to be typically $\lesssim 12 \%$ of unity. In XSPEC our model is:

CONSTANT $\times$ TBABS $_{\text {GAL }} \times[$ ZTBABS $\times$ CABS $\times$ CUTOFFPL + APEC + PEXRAV + ZGAUSS $+f_{\text {SCATT }} \times$ CUTOFFPL $]$.

The results of the spectral fitting are reported in Table 2. In both Observations 5 and 6 we found the source in a Comptonthin state, with a photon index of $\Gamma \sim 1.9$ and a high-energy cutoff of $E_{\mathrm{c}} \gtrsim 200 \mathrm{keV}$. The two observations show a change in $N_{\mathrm{H}}$ (Figure 2), with Observation 5 being less obscured than Observation $6\left(\Delta N_{\mathrm{H}} \sim 10^{23} \mathrm{~cm}^{-2}\right.$, significant at a $\sim 2 \sigma$ level $)$. The two observations also show evidence of intrinsic flux variation, with the source being significantly brighter in Observation 5. The reflection component appears to be rather weak, with a value of $R=0.14_{-0.13}^{+0.16}$ in Observation 6, while it is less constrained in Observation 5 due to the higher flux level. The fraction of scattered flux is found to be $f_{\text {scatt }} \sim 0.5 \%$ of the primary X-ray flux. The X-ray spectra and the ratio between the data and the best-fitting model are shown in Figure 1.

\subsection{Observation 2, 3, and 4}

To study Observations 2, 3, and 4, we set all the parameters, with the exception of the normalization of the primary X-ray emission and the column density, to the values obtained from the study of Observation 6 . The normalization and $N_{\mathrm{H}}$ were allowed to vary within the uncertainties of the values obtained by fitting the X-ray spectrum of Observation 6 . Observation 6 was chosen because the X-ray source was caught in a low-flux state, which allows better constraints on the normalization of the $\mathrm{Fe} \mathrm{K} \alpha$ line and of the reprocessed X-ray emission.

Fitting Observation 2 with the approach described above, we obtained a chi-squared of 49.5 for 28 degrees of freedom (dof), and a clear excess below $6 \mathrm{keV}$. This excess can be removed by 
Table 2

X-Ray Spectral Analysis-Slab Model

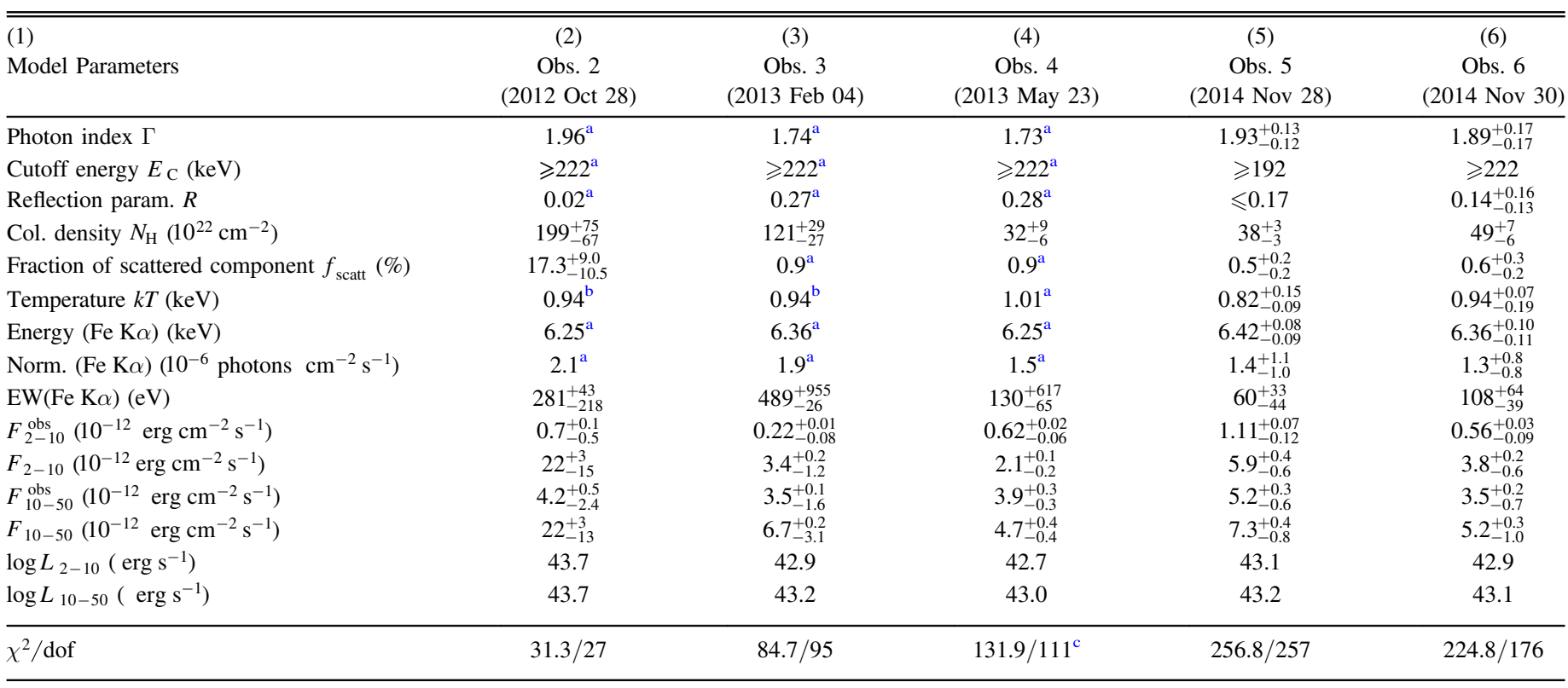

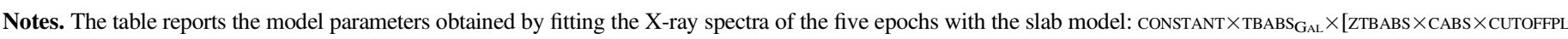

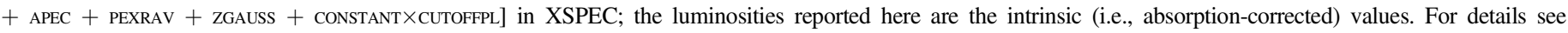
Section 3.1.

${ }^{a}$ Value of the parameter left free to vary within the uncertainties of Observation 6.

b Value of the parameter fixed.

${ }^{c}$ Poissonian statistics applied for Swift/XRT dta. The uncertainties reported for the Fe K $\alpha$ EW correspond to the $68 \%$ confidence interval.

allowing the obscuring material to partially cover the X-ray source, so that some of the primary X-ray flux is able to leak out unabsorbed; i.e., by leaving $f_{\text {scatt }}$ free to vary. This model yields a good value of chi-squared $\left(\chi^{2} /\right.$ dof $\left.=31.3 / 27\right)$, and a fraction of unabsorbed flux of $\sim 17 \%$. Assuming that the scattered fraction is $\sim 0.5 \%$, as found by the spectral analysis of Observations 5 and 6 , this would imply that the absorber is covering $\sim 83 \%$ of the X-ray source in the line of sight. The column density is significantly larger than in Observations 5 and 6, with the obscurer consistent with being CT $\left[\log \left(N_{\mathrm{H}} / \mathrm{cm}^{-2}\right)=24.3\right]$, while the source is in a high-flux state during this observation (Table 2).

The X-ray spectrum of Observation 3 shows that the X-ray source was also obscured by $\mathrm{CT}$ material $\sim 3$ months later $\left[\log \left(N_{\mathrm{H}} / \mathrm{cm}^{-2}\right) \sim 24.13\right]$. As can be seen in Figure 1 , the source was no longer in a high-flux state, and the spectrum shows a prominent $\mathrm{Fe} \mathrm{K} \alpha$ line $(\mathrm{EW} \sim 500 \mathrm{eV})$. Leaving the value of $f_{\text {scatt }}$ free to vary does not significantly improve the chi-squared ( $\Delta \chi^{2} \sim 1$ for 1 less dof). The Fe $\mathrm{K} \alpha \mathrm{EW}$ is higher in Observation 3 than in Observation 2 due to the higher flux level of the X-ray source during Observation 2.

The broadband Swift/XRT-NuSTAR spectrum of Observation 4 shows that IC 751 was in a Compton-thin state at the end of 2013 May, while the X-ray source was significantly dimmer with respect to the previous two observations, with its intrinsic flux level comparable to that observed during Observation 6 .

\section{X-RAY SPECTRAL ANALYSIS-TORUS MODEL}

To further investigate the structure of the absorbers, and to disentangle the torus absorption from that caused by clouds in

\footnotetext{
19 http://www.mytorus.com/
}

the line of sight, assuming an homogeneous torus, we used the MYTorus model $^{19}$ (Murphy \& Yaqoob 2009). The MYTorus model considers absorbed and reprocessed X-ray emission from a smooth torus with a half-opening angle $\theta_{\mathrm{OA}}$ of $60^{\circ}$, and can be used for spectral fitting as a combination of three additive and exponential table models. These tabulated models include the zeroth-order continuum ${ }^{20}$ (MYTORUSZ), the scattered continuum (MYTORUSS), and a component which contains the fluorescent emission lines (MYTORUSL).

\subsection{Standard MYTorus}

The analysis we carried out using the slab model (Section 3) showed that $N_{\mathrm{H}}$ is highly variable, so that it cannot be associated with a smooth absorber alone. This is also confirmed by the fact that applying the smooth MYTorus model to all the $\mathrm{X}$-ray spectra available, setting the values to be the same for the different observations, results in a chi-squared of $\chi^{2}=2628.2$ for 707 dof. Also considering different normalizations of the direct and scattered component or different values of the column density of the scattering and absorbing material fails to reproduce the X-ray spectrum, resulting in values of the reduced chi-squared of $\chi_{\nu}^{2}>2$. We therefore used an alternative approach to take into account variable absorption by combining the non-varying torus absorption [MYTORUSZ (TOR)] with what we define as the cloud absorption. The geometry of the absorber we assume is shown in Figure 3. We adopted a model which includes the three components of MYToRus plus a collisionally ionized plasma, and power law to reproduce the scattered component, similar to what was done

\footnotetext{
${ }^{20}$ This component takes into account both Compton scattering and photoelectric absorption.
} 

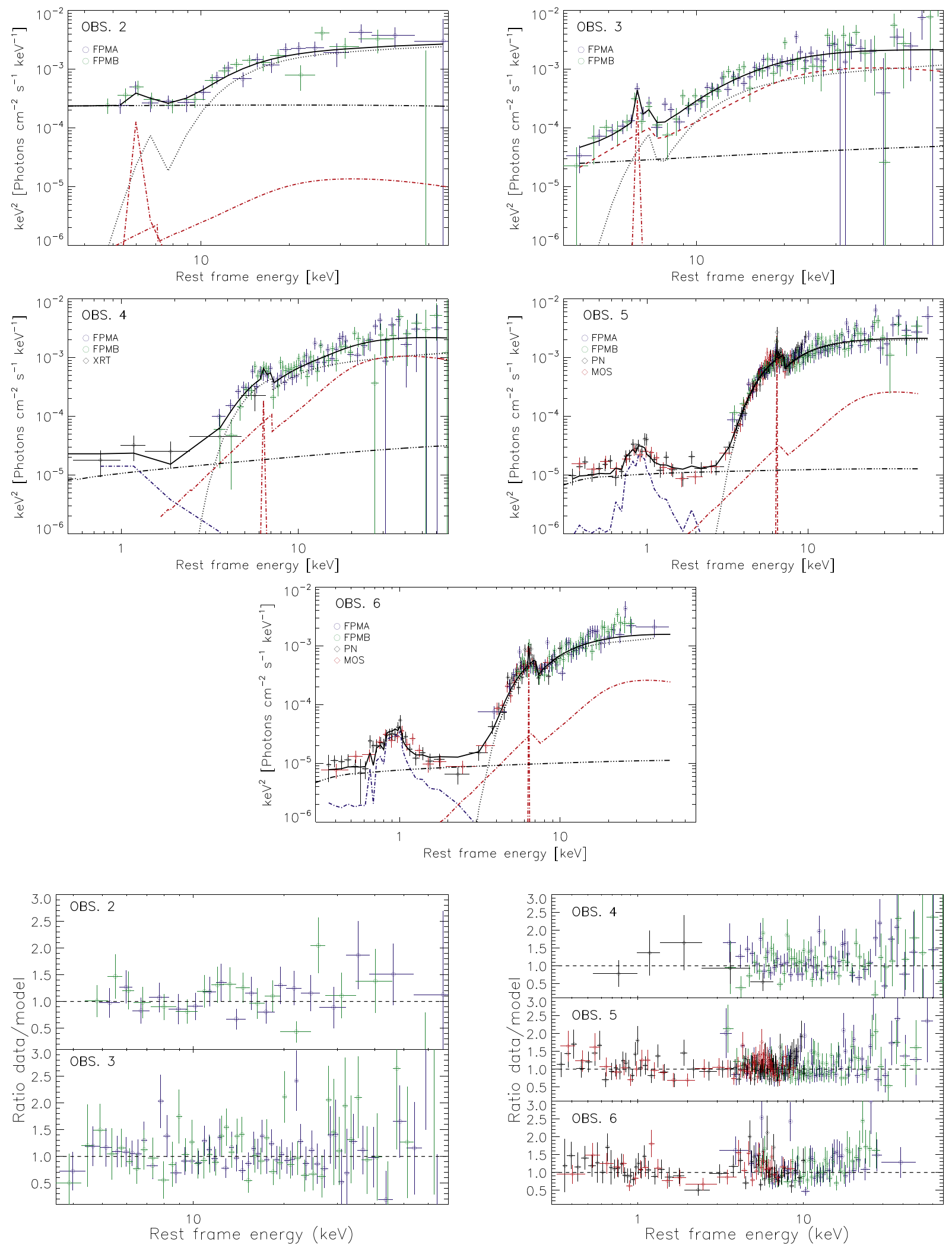

Figure 1. X-ray spectra of IC 751 obtained for the five observations discussed here. Swift/XRT data were rebinned to have a significance of at least $2 \sigma$ per bin only for visual clarity. The black continuous line represents the best fit to the data obtained using the slab model described in Section 3.1. The black dotted line represents the absorbed primary cutoff power-law continuum, the red dotted-dashed line shows the features arising from reprocessed X-ray emission (Compton hump and Fe K $\alpha$ line), the blue dotted-dashed line represents the collisionally ionized plasma emission, while the dotted-dotted-dashed black line is the scattered component. The bottom panels show the ratio between the data and the model obtained for the five observations (symbols are the same as for the X-ray spectra). 


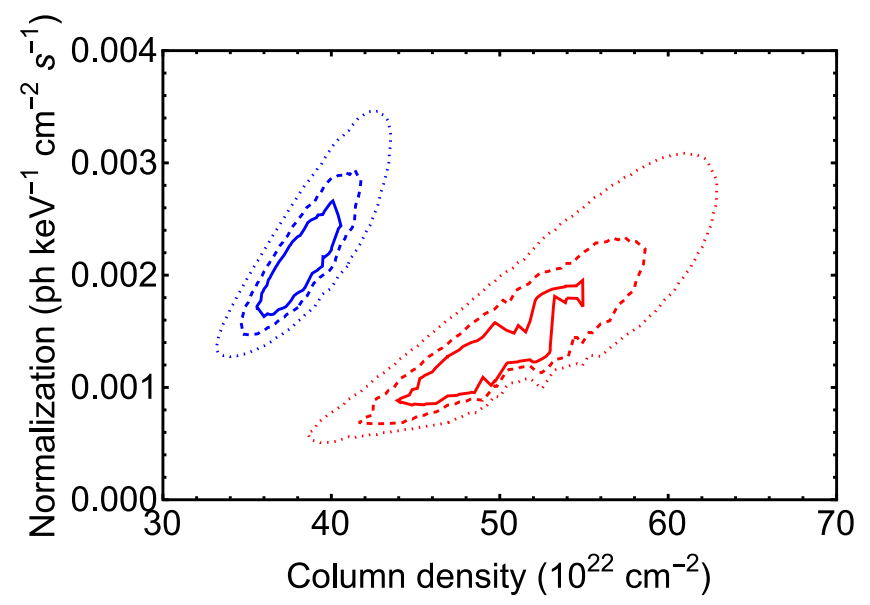

Figure 2. Contour plot of the power-law normalization and the column density for Observations 5 (blue) and 6 (red). Continuous, dashed, and dotted contours represent the $68 \%, 90 \%$, and $99 \%$ confidence intervals, respectively.

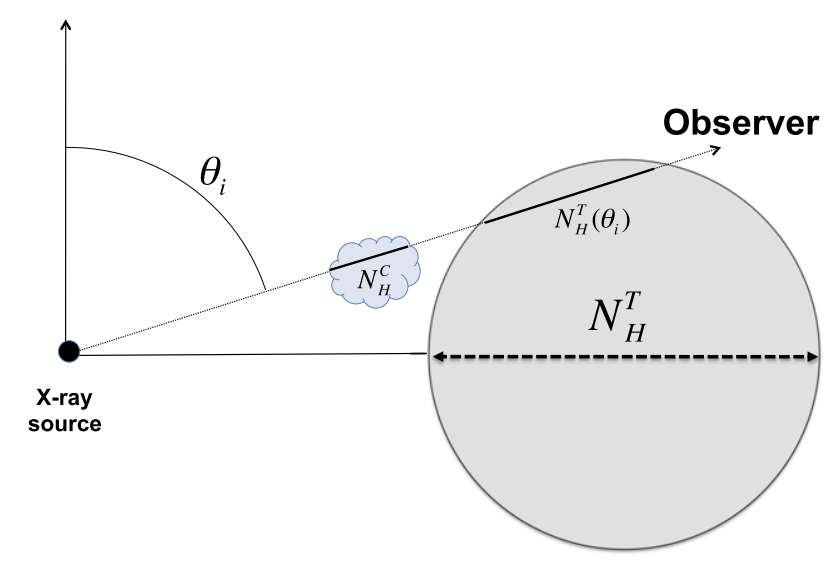

Figure 3. Geometry assumed for the torus model reported in Section 4.1. The parameters $\theta_{\mathrm{i}}, N_{\mathrm{H}}^{\mathrm{C}}, N_{\mathrm{H}}^{\mathrm{T}}\left(\theta_{\mathrm{i}}\right)$, and $N_{\mathrm{H}}^{\mathrm{T}}$ are the inclination angle, the column density of the cloud (variable), the column density of the torus at an angle $\theta_{\mathrm{i}}$ (non-variable), and the equatorial (i.e., maximum) column density of the torus, respectively.

in Section 3. In order to take into account the variable absorber, we used an additional obscuring multiplicative component [MYtorusZ(CLOUd, $N_{\mathrm{H}}^{\mathrm{C}}$ )], where $N_{\mathrm{H}}^{\mathrm{C}}$ is the column density of the cloud. In XSPEC the syntax of our model is:

CONSTANT $\times$ TBABS $_{\text {GAL }} \times[$ MYTORUSZ(CLOUD) $\times$ MYTORUSZ

$($ TOR $) \times$ ZPOWERLAW + MYTORUSS + APEC + GSMOOTH(MYTORUSL) $+f_{\text {scatt }} \times$ ZPOWERLAW].

The free parameters of MYTorus are the photon index $\Gamma$, the equatorial column density of the torus $N_{\mathrm{H}}^{\mathrm{T}}$, and the inclination angle of the observer $\theta_{\mathrm{i}}$. We convolved the fluorescent emission lines of MYTorus using a Gaussian function (GSMOOTH in XSPEC) to take into account the expected velocity broadening. We fixed the width of the lines to a FWHM of $2000 \mathrm{~km} \mathrm{~s}^{-1}$, consistent with the average value obtained for 36 AGNs at $z<0.3$ by the Chandra/HEG study of Shu et al. (2010). The model was also multiplied by a constant to take into account cross-calibration between the different spectra.

We simultaneously fitted the five sets of observations discussed above. The values of $\theta_{\mathrm{i}}, N_{\mathrm{H}}^{\mathrm{T}}, \Gamma$, and the normalization of the scattered component $\left(n_{\text {refl }}\right.$, which includes the Compton hump) were left free to vary, and tied to be constant for all observations. The normalization of the fluorescent lines was fixed to $n_{\text {refl }}$. The normalization of the primary power-law component $\left(n_{\text {po }}\right)$, the value of $N_{\mathrm{H}}^{\mathrm{C}}$, and that of $f_{\text {scatt }}$ were left free to have independent values for different observations.

The results obtained by this torus model are reported in the upper part of Table 3. The fit yields a chi-squared of $\chi^{2}=774.8$ (for $688 \mathrm{dof}$ ), and the primary X-ray emission has a value of the photon index consistent with that found using the slab model. As with the slab model, we find a clear variation of the line of sight column density, with the clouds having values of the column density spanning between $1.5 \times 10^{24}$ and $5 \times 10^{21} \mathrm{~cm}^{-2}$. This approach also confirms a significant change in the value of $f_{\text {scatt }}$ between the five observations, which varies between $\sim 4 \%$ and $\sim 0.4 \%$. These variations in $f_{\text {scatt }}$ are interpreted as being due to a partially covering absorber in the line of sight. Contrary to what we obtained using the slab model, applying MYToRus we do not find a significant variation of the line of sight column density between Observation 5 and Observation 6. The model used, with the parameters set to those obtained for Observation 2, is shown in Figure 4.

We found an equatorial column density of $N_{\mathrm{H}}^{\mathrm{T}}$ $\simeq 4.8 \times 10^{24} \mathrm{~cm}^{-2}$ and an inclination angle of the observer of $\theta_{i} \simeq 60^{\circ} 3$, close to grazing incidence. The line of sight column density in the toroidal geometry assumed by MYTORUS can be obtained by

$$
N_{\mathrm{H}}^{\mathrm{T}}\left(\theta_{\mathrm{i}}\right)=N_{\mathrm{H}}^{\mathrm{T}}\left(1-4 \cos ^{2} \theta_{\mathrm{i}}\right)^{\frac{1}{2}},
$$

which implies that for IC $751 N_{\mathrm{H}}^{\mathrm{T}}\left(\theta_{\mathrm{i}}\right) \simeq 6.4 \times 10^{23} \mathrm{~cm}^{-2}$, a value larger than the lowest column density inferred by adopting the slab model. However, given the dependence of the column density on the inclination angle, and the problems associated with the toroidal geometry for inclination angles close to the edges (see discussion in Yaqoob 2012), the uncertainty associated with this value is large.

\subsection{Decoupled MYTorus}

To further test the structure of the absorber, we applied MYTorus in the decoupled mode (Yaqoob 2012). This was done by: (i) separating the column density of the absorbing $\left[N_{\mathrm{H}}^{\mathrm{T}}(Z)\right]$ and reprocessing $\left[N_{\mathrm{H}}^{\mathrm{T}}(S, L)\right]$ material, leaving both of them free to vary; (ii) fixing the inclination angle of MYTORUSL and MYTORUSS to $\theta_{\mathrm{i}}(S, L)=0^{\circ}$, and that of MYTORUSZ to $\theta_{\mathrm{i}}(Z)=90^{\circ}$; (iii) adding a second scattered component with $\theta_{\mathrm{i}}(S, L)=90^{\circ}$ and the same column density and normalization of the one with $\theta_{\mathrm{i}}(S, L)=0^{\circ}$; (iv) leaving the normalizations of the two components ( $n_{\text {po }}$ and $n_{\text {refl }}$ ) free, as was done in Section 4.1. The model we used assumes a geometry which consists of two absorbers, one varying and one constant with time, plus reprocessing material with a different value of the column density.

The results obtained are reported in the lower part of Table 3. The decoupled MYTorus model yields a better chi-squared $\left(\chi^{2}=749.2\right)$ than the non-decoupled one, for the same number of dof. The values of $f_{\text {scatt }}$ are consistent with those found by applying the standard MYTonus model, while the power-law continuum is slightly steeper. With this model we find a significant variation of $N_{\mathrm{H}}^{\mathrm{C}}$ between Observations 5 and 6 , similar to what we found using PEXRAV. 
Table 3

X-Ray Spectral Analysis-Torus Model

\begin{tabular}{|c|c|c|c|c|c|c|c|c|}
\hline \multicolumn{9}{|c|}{ MYTorus } \\
\hline (1) & (2) & (3) & (4) & (5) & (6) & (7) & (8) & (9) \\
\hline Observation & $\begin{array}{c}N_{\mathrm{H}}^{\mathrm{C}} \\
\left(10^{22} \mathrm{~cm}^{-2}\right)\end{array}$ & $\begin{array}{c}n_{\mathrm{po}} \\
\left(10^{-3} \mathrm{ph} \mathrm{keV}^{-1} \mathrm{~cm}^{-2} \mathrm{~s}^{-1}\right)\end{array}$ & $\Gamma$ & $\begin{array}{c}N_{\mathrm{H}}^{\mathrm{T}} \\
\left(10^{24} \mathrm{~cm}^{-2}\right)\end{array}$ & $\begin{array}{c}\theta_{\mathrm{i}} \\
(\mathrm{deg})\end{array}$ & $\begin{array}{c}n_{\text {refl }} \\
\left(10^{-3} \mathrm{ph} \mathrm{keV}^{-1} \mathrm{~cm}^{-2} \mathrm{~s}^{-1}\right)\end{array}$ & $\begin{array}{c}\mathrm{kT} \\
(\mathrm{keV})\end{array}$ & $\begin{array}{l}f_{\text {scatt }} \\
(\%)\end{array}$ \\
\hline $2[2012.82]$ & $150_{-15}^{+19}$ & $3.83_{-0.42}^{+0.43}$ & $1.88_{-0.04}^{+0.01}$ & $4.76_{-0.27}^{+0.09}$ & $60.3_{-0.2}^{+0.2}$ & $1.14_{-0.11}^{+0.15}$ & $0.93_{-0.04}^{+0.04}$ & $4.2_{-0.8}^{+0.8}$ \\
\hline 3 [2013.10] & $111_{-7}^{+8}$ & $2.10_{-0.17}^{+0.18}$ & // & // & // & // & // & $1.6_{-0.5}^{+0.5}$ \\
\hline 4 [2013.39] & $5.1_{-3.1}^{+3.0}$ & $0.94_{-0.06}^{+0.06}$ & // & // & // & // & // & $3.2_{-1.2}^{+1.5}$ \\
\hline \multicolumn{9}{|c|}{ MYTorus_-Decoupled Mode } \\
\hline Observation & $\begin{array}{c}N_{\mathrm{H}}^{\mathrm{C}} \\
\left(10^{22} \mathrm{~cm}^{-2}\right)\end{array}$ & $\begin{array}{c}n_{\mathrm{po}} \\
\left(10^{-3} \mathrm{ph} \mathrm{keV}^{-1} \mathrm{~cm}^{-2} \mathrm{~s}^{-1}\right)\end{array}$ & $\Gamma$ & $\begin{array}{c}N_{\mathrm{H}}^{\mathrm{T}}(Z) \\
\left(10^{24} \mathrm{~cm}^{-2}\right)\end{array}$ & $\begin{array}{c}N_{\mathrm{H}}^{\mathrm{T}}(S, L) \\
\left(10^{24} \mathrm{~cm}^{-2}\right)\end{array}$ & $\begin{array}{c}n_{\text {refl }} \\
\left(10^{-3} \mathrm{ph} \mathrm{keV}^{-1} \mathrm{~cm}^{-2} \mathrm{~s}^{-1}\right)\end{array}$ & $\begin{array}{c}\mathrm{kT} \\
(\mathrm{keV})\end{array}$ & $\begin{array}{c}f_{\text {scatt }} \\
(\%)\end{array}$ \\
\hline 2 [2012.82] & $147_{-48}^{+82}$ & $4.71_{-2.45}^{+6.29}$ & $1.98_{-0.07}^{+0.08}$ & $0.37_{-0.02}^{+0.02}$ & $6.0_{-2.2}^{+3.5}$ & $1.22_{-0.36}^{+0.50}$ & $0.93_{-0.11}^{+0.05}$ & $4.1_{-2.0}^{+3.4}$ \\
\hline 6 [2014.92] & $11.1_{-4.9}^{+5.0}$ & $1.28_{-0.27}^{+0.38}$ & // & // & // & // & // & $0.6_{-0.3}^{+0.2}$ \\
\hline
\end{tabular}

Note. Model parameters obtained by simultaneously fitting the 15 X-ray spectra of IC 751 (divided in five epochs). The table reports (1) the observation number, (2) the cloud column density, (3) the normalization of the primary power-law emission, (4) the photon index of the primary X-ray emission, (5) the equatorial column density of the torus, (6) the inclination angle of the observer (see Figure 3), (7) the normalization of the reflection component, (8) the temperature of the collisionally ionized plasma, and (9) the fraction of scattered unabsorbed emission. The upper part of the table refers to the MYToRus model in his original formulation (with the addition of a cloud of neutral material; see Section 4.1), while the lower part reports the results obtained by using MYToRus in the decoupled mode (Section 4.2). In the lower part column (5) is the torus column density of the MYTORuSZ component for $\theta_{\mathrm{i}}(Z)=90^{\circ}$, and (6) is the column density of the MYTORUSS and MYTORUsL components, assuming $\theta_{\mathrm{i}}(S, L)=0^{\circ}$ and $\theta_{\mathrm{i}}(S, L)=90^{\circ}$.

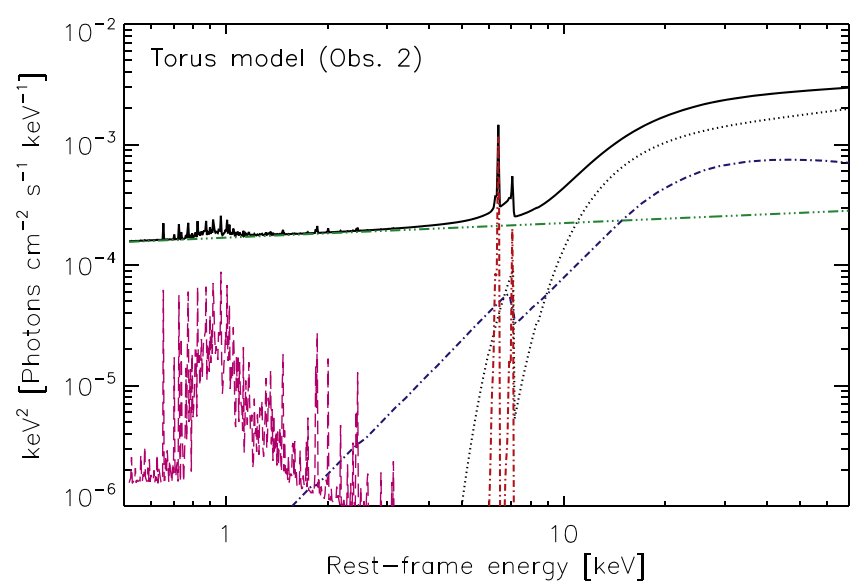

Figure 4. Torus spectral model used for the analysis of IC 751. The black continuous line represents the total flux, while the components shown are the absorbed X-ray power-law (ZPOW $\times$ ZTBABS $\times$ CABS $\times$ MYTORUSZ, dotted black line), the scattered component from the torus (MYTORuSS, dotted-dashed blue line), the fluorescent emission lines (MYTORusL, dotted-dashed red line), the thermal component (magenta dashed line), and the scattered emission (green dotted-dotted-dashed line). The values are set to those obtained during Observation 2. For more details, see Section 4.1 and Table 3.

The column density of the reprocessing material is found to be $N_{\mathrm{H}}^{\mathrm{T}}(S, L)=\left(6.0_{-2.2}^{+3.5}\right) \times 10^{24} \mathrm{~cm}^{-2}$, while the line of sight non-variable absorber $\left[N_{\mathrm{H}}^{\mathrm{T}}(Z)\right]$ has a lower value $\left(\sim 3.7 \times 10^{23} \mathrm{~cm}^{-2}\right)$ than that obtained considering a homogeneous torus (Section 4.1). The value of $N_{\mathrm{H}}^{\mathrm{T}}(Z)$ is consistent with the lowest value of $N_{\mathrm{H}}$ obtained by applying the slab model.

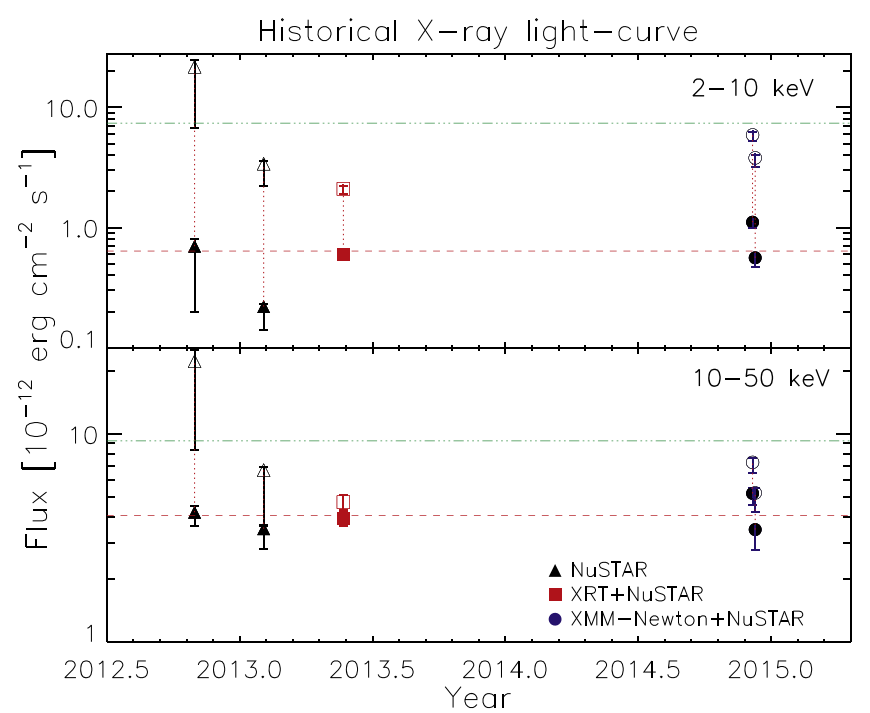

Figure 5. Historical X-ray variability of IC 751 in the 2-10 (top panel) and $10-50 \mathrm{keV}$ (bottom panel) band. The filled and empty points represent the observed and intrinsic (i.e., corrected for absorption) fluxes, respectively. The red dashed line and the dotted-dotted-dashed green line represent the average observed and intrinsic fluxes, respectively.

\section{FLUX VARIABILITY AND TIME-RESOLVED SPECTROSCOPY}

X-ray observations have shown that besides a highly variable line of sight column density, IC 751 also presents significant flux variability of the primary $\mathrm{X}$-ray source on timescales of days to months both in the soft and hard X-ray bands (see Tables 2 and 3). As illustrated in Figure 5 (filled 
points), the observed flux of IC 751 varies by a factor of 4 in the $2-10 \mathrm{keV}$ band, and by a factor of 1.6 in the $10-50 \mathrm{keV}$ band. When considering intrinsic fluxes (i.e., absorptioncorrected, empty points in Figure 5), the amplitude of the variability is larger: the $\mathrm{X}$-ray source varies by a factor of $\sim 5$ both in the $2-10$ and $10-50 \mathrm{keV}$ bands. The average observed fluxes in the $2-10 \mathrm{keV}$ and $10-50 \mathrm{keV}$ bands are $6.4 \times 10^{-13} \mathrm{erg} \mathrm{cm}^{-2} \mathrm{~s}^{-1}$ and $4.0 \times 10^{-12} \mathrm{erg} \mathrm{cm}^{-2} \mathrm{~s}^{-1}$, respectively. The intrinsic average nuclear fluxes in the two bands are $7.4 \times 10^{-12} \mathrm{erg} \mathrm{cm}^{-2} \mathrm{~s}^{-1}(2-10 \mathrm{keV})$ and $9.3 \times 10^{-12} \mathrm{erg} \mathrm{cm}^{-2} \mathrm{~s}^{-1}(10-50 \mathrm{keV})$, which correspond to k-corrected average luminosities of $\log \left(L_{2-10} / \mathrm{erg} \mathrm{s}^{-1}\right)$ $=43.22$ and $\log \left(L_{10-50} / \mathrm{erg} \mathrm{s}^{-1}\right)=43.32$. The $12 \mu \mathrm{m}$ rest frame luminosity of IC 751 could be estimated using WISE by linearly interpolating the fluxes in the $W 3(11.56 \mu \mathrm{m})$ and $W 4$ $(22.09 \mu \mathrm{m})$ bands. The mid-IR flux of IC 751 is dominated by the AGNs, since $W 1-W 2>0.8$ (Stern et al. 2012). We found that the X-ray luminosity is in agreement with the $12 \mu \mathrm{m}$ luminosity $\left[\log \left(L_{12 \mu \mathrm{m}} / \mathrm{erg} \mathrm{s}^{-1}\right)=43.70\right]$, as expected from the well known mid-IR/X-ray correlation (e.g., Gandhi et al. 2009, Asmus et al. 2015, Stern 2015).

In order to improve our constraints on the absorbing material, and to study its evolution on shorter timescales, we analyzed the XMM-Newton EPIC/PN and NuSTAR light curves of IC 751 in different energy bands. We extracted XMMNewton EPIC/PN light curves in the 0.3-10, 0.3-2, and $2-10 \mathrm{keV}$ bands, with bins of $1 \mathrm{ks}$. NuSTAR FPMA light curves were extracted in the 3-79, 3-20, and 20-60 keV bands with bins of $6 \mathrm{ks}$. We also analyzed the variability of the hardness ratio, defined as $H R=H-S / H+S$, where $H$ and $S$ are the fluxes in the soft $(0.3-2$ and $3-20 \mathrm{keV})$ and hard (2-10 and 20-60 keV) bands, respectively.

For all the observations we performed a $\chi^{2}$ test in order to assess the variability of the hardness ratio and the flux in the three different energy bands. We considered the flux or the hardness ratio to be variable if the minimum confidence level was $p \leqslant 1 \%$. To constrain the amplitude of the variability, we used the rms variability amplitude $\left(F_{\text {var }}\right.$; see Equation $(10)$ and B2 of Vaughan et al. 2003). NuSTAR light curves of Observation 2 show significant variability both in the broad and in the soft bands, while the other NuSTAR observations do not show any sign of short-term variability. The XMM-Newton light curve of Observation 5 shows significant flux variation in the hard band, while the flux is consistent with being constant during Observation 6. The largest value of the rms variability amplitude is found for the NUSTAR 3-20 keV light curve of Observation $2 \quad\left(F_{\mathrm{var}}=34 \pm 7 \%\right)$. The hardness ratio is significantly variable only for the NUSTAR light curve of Observation 2 ( $\left.p \simeq 0.5 \%, F_{\text {var }}=59 \pm 16 \%\right)$, which shows a hardening of the spectrum in the last $\sim 6 \mathrm{ks}$ of the observation.

The 104 month 14-195 Swift/BAT light curve does not show evidence of significant long-term variability $(p \sim 6 \%)$ on a timescale of $25 \mathrm{Ms}$.

We also carried out a time-resolved spectral analysis of the longest NUSTAR observation (Observation 3) by splitting it in five time intervals with similar lengths. We applied the slab model described in Section 3, leaving the normalization of the primary X-ray emission and the line of sight column density free to vary, and found that the parameters obtained are consistent within their $90 \%$ confidence interval between all observations. Leaving the value of $f_{\text {scatt }}$ free to vary significantly improves the fit only for the second $\left(\Delta \chi^{2} \simeq 8\right)$ and third

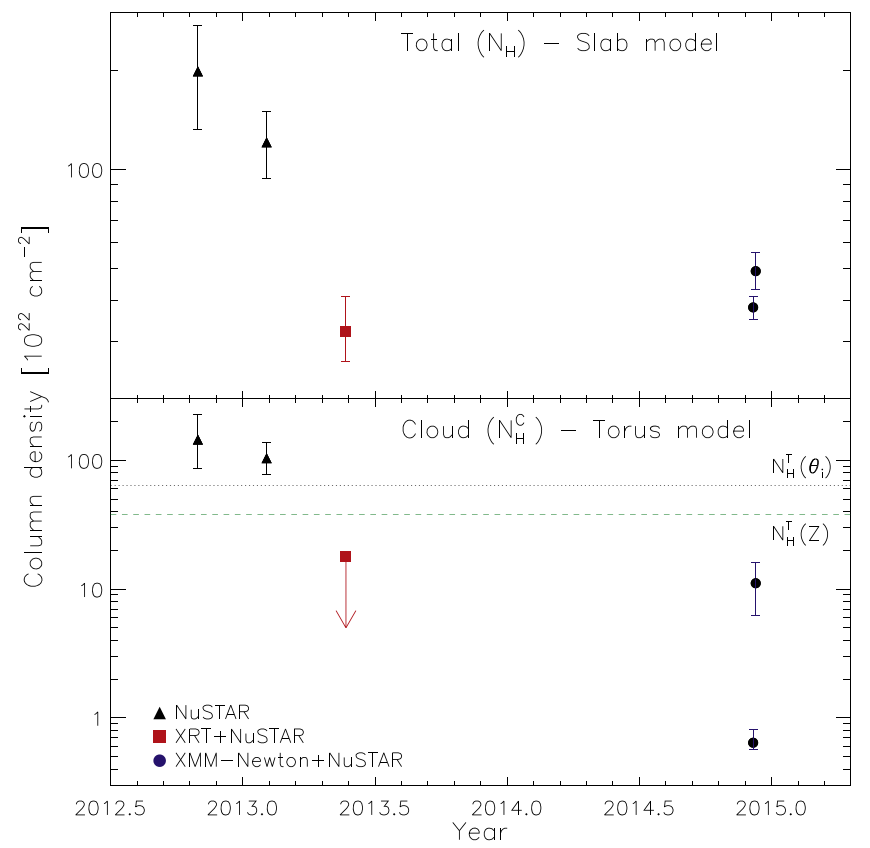

Figure 6. Column density variability of IC 751. Top panel: total line of sight column density $\left(N_{\mathrm{H}}\right)$ obtained with the slab model described in Section 3. Bottom panel: column density of the varying absorber (cloud column density, $N_{\mathrm{H}}^{\mathrm{C}}$; see Figure 3) obtained with the torus spectral model discussed in Section 4. The black dotted line and the green dashed line represent the values of the non-variable column density of the torus obtained by using MYToRus in its standard $\left[N_{\mathrm{H}}^{\mathrm{T}}\left(\theta_{\mathrm{i}}\right)\right]$ and decoupled $\left[N_{\mathrm{H}}^{\mathrm{T}}(Z)\right]$ modes, respectively. The values of the line of sight column density are those obtained by using MYToRus in the decoupled mode (Section 4.2).

$\left(\Delta \chi^{2} \simeq 7\right)$ segments, and results in values consistent with those obtained for the other segments and for the whole observation.

\section{DISCUSSION}

The X-ray observations of IC 751 presented here show clear evidence of changes in the line of sight column density, with the X-ray source being obscured by Compton-thick $\left[\log \left(N_{\mathrm{H}} / \mathrm{cm}^{-2}\right) \sim 24.3\right]$ material in two observations, and by Compton-thin material $\left[\log \left(N_{\mathrm{H}} / \mathrm{cm}^{-2}\right) \simeq 23.60\right]$ in three observations. This result is confirmed adopting both the slab (Section 3, top panel of Figure 6) and the torus (Section 4, bottom panel of Figure 6) X-ray spectral models. In particular, by using a physical torus model, we were able, assuming a smooth and azimuthally symmetric torus, to disentangle the intrinsic obscuration associated with a non-varying absorber from the column density of the varying absorber. Variations in the observed line of sight column density might be related either to: (i) intrinsic variation of the absorbing material, caused by moving clouds; (ii) changes in the intensity of the nuclear radiation, which would cause a variation in the ionization state of the absorbing material. Although the observations during which the highest values of the column density were also found to correspond to the stage in which the source was more luminous, for a low-density photo-ionized absorber there might be delay between the flux variation and the response of the absorber, so that the second scenario cannot be completely discarded. In the following, we will, however, assume that changes in $N_{\mathrm{H}}$ are related to clouds eclipsing the X-ray source, 
as found for several other changing look AGNs (e.g., Risaliti et al. 2005).

Following Risaliti et al. (2007) and Marinucci et al. (2013), the distance of the cloud from the X-ray source $\left(R_{c}\right)$ can be estimated by considering that the size of the source and that of the cloud are similar $\left(D_{c} \simeq D_{\mathrm{s}}\right)$, and that the transverse velocity is given by the ratio between the size of the source $\left(D_{s}\right)$ and the crossing time $T_{\mathrm{cr}}: V_{k}=D_{s} / T_{\mathrm{cr}}$. It must be remarked that in Observation 2 we found possible evidence of partial covering, which would imply that $D_{c}<D_{s}$. By applying the slab model, we found that the cloud covers $83 \%$ of the X-ray source. The value of the covering factor was larger when adopting the torus model $(\sim 96 \%)$. Given the rather large values of the covering factor, taking the possible difference between $D_{c}$ and $D_{s}$ into account does not significantly affect our results. Assuming that the cloud is moving with a Keplerian velocity, we obtain:

$$
R_{c}=\frac{G M_{\mathrm{BH}}}{V_{k}^{2}}=\frac{G M_{\mathrm{BH}} T_{\mathrm{cr}}^{2}}{D_{s}^{2}} .
$$

Micro-lensing (e.g., Chartas et al. 2002, 2009), occultation studies (Risaliti et al. 2009), and large-amplitude rapid X-ray variability have shown that the size of the X-ray source is $D_{\mathrm{s}} \simeq 10 r_{\mathrm{g}}$, where $r_{\mathrm{g}}=G M_{\mathrm{BH}} / c^{2}$. Assuming that $D_{\mathrm{s}}=10 r_{\mathrm{g}}$, we obtain

$$
R_{c}=\frac{G M_{\mathrm{BH}} T_{\mathrm{cr}}^{2}}{10^{2} R_{\mathrm{G}}^{2}} \simeq 2 \mathrm{pc} M_{8.5} R_{10}^{-2} T_{10}^{2},
$$

where $T_{10}$ is the crossing time in units of 10 days $\left(8.64 \times 10^{5}\right.$ s) and $M_{8.5}=M_{\mathrm{BH}} / 10^{8.5} M_{\odot}$.

The black hole mass of IC 751 has recently been obtained by the study of the stellar velocity dispersion as part of work aimed at constraining the characteristics of Swift/BAT-selected AGNs in the optical band (M. J. Koss et al. 2016, in preparation), and is $\log \left(M_{\mathrm{BH}} / M_{\odot}\right) \simeq 8.5$. By using Equation (3), and considering that (i) no significant variation was found during the $50 \mathrm{ks}$ of NuSTAR Observation 3 (over a total of $100 \mathrm{ks}$ ), and that (ii) the shortest interval in which a variation of the CT material is evident is between Observation 3 and Observation 4, which were carried out 108 days apart, we can say that the distance of the cloud is between $R_{\min }=0.027 \mathrm{pc}$ ( $\sim 32$ light days) and $R_{\max } \simeq 230$ pc.

Optical reverberation-mapping studies have shown that the radius of the BLR scales with the square root of the luminosity (e.g., Kaspi et al. 2005). According to Kaspi et al. (2005), considering the $\mathrm{H} \beta$ lags and the results obtained averaging different observations of the same object, the radius of the BLR is given by

$$
\frac{R_{\mathrm{BLR}}}{10 \mathrm{lt}-\text { days }}=0.86 \times\left(\frac{L_{2-10}}{10^{43} \mathrm{erg} \mathrm{s}^{-1}}\right)^{0.532} .
$$

For the average $2-10 \mathrm{keV}$ luminosity of IC 751, we find $R_{\mathrm{BLR}}=11.7$ light days, which implies that the obscuring clouds responsible for the variation of $N_{\mathrm{H}}$ are beyond the emission-weighted average radius of the BLR. Using a similar approach, it is possible to put constraints on the location of the hot inner wall of the torus, which is also known to scale with the square root of the luminosity (e.g., Suganuma et al. 2006, Kishimoto et al. 2011). Following Tristram \& Schartmann 2011 (Figure 4 of their paper), the inner radius of the hot dust $\left(R_{\mathrm{NIR}}\right)$, obtained from $K$-band reverberation, can be approximated by:

$$
\log \frac{R_{\mathrm{NIR}}}{1 \mathrm{pc}}=-23.10+0.5 \log L_{14-195},
$$

where $L_{14-195}$ is the $14-195 \mathrm{keV}$ luminosity (in $\mathrm{erg} \mathrm{s}^{-1}$ ). At $12 \mu \mathrm{m}$, interferometric studies (Tristram \& Schartmann 2011; see also Burtscher et al. 2013) have shown that the size of the mid-IR-emitting region $\left(R_{\mathrm{MIR}}\right)$ in AGNs can be estimated by

$$
\log \frac{R_{\mathrm{MIR}}}{1 \mathrm{pc}}=-21.62+0.5 \log L_{14-195 .}
$$

The 70 month averaged $14-195 \mathrm{keV}$ luminosity of IC 751 is $\log \left(L_{14-195} / \mathrm{ergs}^{-1}\right)=43.47$ (Baumgartner et al. 2013), which corresponds to $R_{\mathrm{NIR}}=0.04 \mathrm{pc} \quad(\sim 48$ light days $)$ and $R_{\mathrm{MIR}}=1.3 \mathrm{pc}$. From this we can conclude that the absorbing material could be related to the outer BLR, to clumps in the molecular torus, or even to material located at a further distance from the SMBH.

A change in the Compton-thin absorber is also found between Observation 5 and Observation 6 using the slab spectral model and MYTORUS in its decoupled mode. The two observations were carried out about $48 \mathrm{hr}$ apart, which means (applying Equation (3)) that for the Compton-thin material the $R \lesssim R_{\max }=0.08 \mathrm{pc} \quad(\sim 95$ light days). If the Compton-thin and CT clouds are located at the same distance from the X-ray source, then the regions where the varying absorbers are located are between 32 and 95 light days. This would imply that the absorber is consistent with being located either in the BLR or in the inner side of the dusty torus. Recent work has shown that the $\mathrm{Fe} \mathrm{K} \alpha$ might also arise in this region (Gandhi et al. 2015; Minezaki \& Matsushita 2015). Assuming that the cloud has about the same size of the X-ray source, i.e., $\sim 10 r_{\mathrm{g}}\left(\sim 4.7 \times 10^{14} \mathrm{~cm}\right)$, and that its column density is $1.5 \times 10^{24} \mathrm{~cm}^{-2}$, the density of the cloud would be $n \sim 3.2 \times 10^{9} \mathrm{~cm}^{-3}$. This value is in agreement with that expected for the BLR clouds (e.g., Peterson 1997). A BLR origin for the varying absorber in IC 751 would fit what has been found so far for other changing look AGNs, several of which show absorbers compatible with being part of the BLR (e.g., Maiolino et al. 2010; Risaliti et al. 2010; Burtscher et al. 2015).

Thanks to its broadband coverage, NuSTAR is a very powerful tool to study obscuration in AGNs, and it has been shown to be fundamental to well constrain the line of sight column density (e.g., Arévalo et al. 2014; Bauer et al. 2015; Gandhi et al. 2014; Annuar et al. 2015; Koss et al. 2015; Lansbury et al. 2015). Studying type II quasars, Lansbury et al. (2015) have shown that the estimates of $N_{\mathrm{H}}$ obtained by NUSTAR can be 2.5-1600 times higher than previous constraints from XMM-Newton and Chandra. This shows that in the absence of high-quality broadband observations, it would be possible to miss changing look events for weak sources. Another clear example is given by the recent detection of an unveiling event in NGC 1068 (Marinucci et al. 2016), which would have been missed by observations carried out below $10 \mathrm{keV}$. Repeated NUSTAR observations of obscured sources might therefore uncover a significant number of new changing look events. Burtscher et al. (2015) have recently reanalyzed the relation between $N_{\mathrm{H}}$ and the optical obscuration $A_{\mathrm{V}}$, and found that in several cases the deviation of $N_{\mathrm{H}} / A_{\mathrm{V}}$ from the Galactic value is due to variable absorption. This 
would imply that ideal targets to study occultations of the X-ray source are objects showing a large deviation from the Galactic $N_{\mathrm{H}} / A_{\mathrm{V}}$ value.

\section{SUMMARY AND CONCLUSIONS}

We reported here on the spectral analysis of five NuSTAR observations of the type 2 AGN IC 751, three of which were combined with XMM-Newton or Swift/XRT observations in the $0.3-10 \mathrm{keV}$ range. IC 751 is the first changing look AGN (i.e., an object that has been observed both in a Compton-thin and a CT state) discovered by NuSTAR. We find that the X-ray source was obscured by CT material during the first two observations, while its line of sight obscuration is found to be Compton-thin during the following observations, which implies that absorption varies on timescales of $\lesssim 3$ months. Changes of the line of sight column density are also found on a timescale of $\sim 48 \mathrm{hr}\left(\Delta N_{\mathrm{H}} \sim 10^{23} \mathrm{~cm}^{-2}\right)$. While we cannot constrain the location of the absorber precisely, by considering the lack of spectral variability during the longest NuSTAR observation we can infer the minimum distance to be further than the emission-weighted average radius of the BLR. Assuming that the varying Compton-thin and CT clouds are located at the same distance from the X-ray source, then the material is located between 32 and 95 light days. The absorber could therefore be related to either the external part of the BLR or to the inner part of the dusty torus, although the BLR origin might be slightly favored since the density of the clouds is found to be consistent with the value expected for BLR clouds. By adopting a physical torus X-ray spectral model, we are able to disentangle the column density of the non-varying absorber $\left(N_{\mathrm{H}} \sim 3.8 \times 10^{23} \mathrm{~cm}^{-2}\right)$ from that of the varying clouds $\left[N_{\mathrm{H}} \sim(1-150) \times 10^{22} \mathrm{~cm}^{-2}\right]$, and to put constraints on the column density of the reprocessing material $\left(N_{\mathrm{H}} \sim\right.$ $\left.6 \times 10^{24} \mathrm{~cm}^{-2}\right)$. We found that the $\mathrm{X}$-ray source is highly variable in both the $2-10 \mathrm{keV}$ and $10-50 \mathrm{keV}$ bands. Future observational campaigns on IC 751 in the X-ray band will be able to improve the constraints on the location of the varying absorber, and confirm or not whether it is related to clouds in the BLR as has been found for several objects of this class.

We thank the anonymous referee for comments that helped us to improve the quality of our manuscript. CR acknowledges Marko Stalevski and Sebastien Guillot for fruitful discussions, and Chin-Shin Chang for her comments on the manuscript. This research has made use of the NuSTAR Data Analysis Software (NuSTARDAS) jointly developed by the ASI Science Data Center (ASDC, Italy) and the California Institute of Technology (Caltech, USA). We acknowledge financial support from the CONICYT-Chile grants "EMBIGGEN" Anillo ACT1101 (CR, ET, FEB, PA), FONDECYT 1141218 (CR, FEB), FONDECYT 1140304 (PA), Basal-CATA PFB06/2007 (CR, ET, FEB), NuSTAR subcontract 44A-1092750 (WNB), the Swiss National Science Foundation and Ambizione fellowship grant PZ00P2 154799/1 (MK), and the Ministry of Economy, Development, and Tourism's Millennium Science Initiative through grant IC120009, awarded to The Millennium Institute of Astrophysics, MAS (FEB). This research has made use of the NASA/IPAC Extragalactic Database (NED) which is operated by the Jet Propulsion Laboratory, of data obtained from the High Energy Astrophysics Science Archive Research Center (HEASARC), provided by NASA's Goddard Space Flight Center, and of the SIMBAD Astronomical Database which is operated by the Centre de Données astronomiques de Strasbourg.

Facilities: NuSTAR, Swift, XMM-Newton.

\section{REFERENCES}

Annuar, A., Gandhi, P., Alexander, D. M., et al. 2015, ApJ, 815, 36 Arévalo, P., Bauer, F. E., Puccetti, S., et al. 2014, ApJ, 791, 81

Arnaud, K. A. 1996, in ASP Conf. Ser. 101, Astronomical Data Analysis Software and Systems V, ed. G. H. Jacoby, \& J. Barnes (San Francisco, CA: ASP), 17

Asmus, D., Gandhi, P., Hoenig, S. F., Smette, A., \& Duschl, W. J. 2015, MNRAS, 454, 766

Bauer, F. E., Arevalo, P., Walton, D. J., et al. 2015, ApJ, 812, 116

Baumgartner, W. H., Tueller, J., Markwardt, C. B., et al. 2013, ApJS, 207, 19

Beckmann, V., Jean, P., Lubiński, P., Soldi, S., \& Terrier, R. 2011, A\&A, 531, A70

Bianchi, S., Guainazzi, M., Matt, G., et al. 2005, A\&A, 442, 185

Bianchi, S., Maiolino, R., \& Risaliti, G. 2012, AdAst, 2012, 17

Bianchi, S., Piconcelli, E., Chiaberge, M., et al. 2009, ApJ, 695, 781

Braito, V., Ballo, L., Reeves, J. N., et al. 2013, MNRAS, 428, 2516

Burrows, D. N., Hill, J. E., Nousek, J. A., et al. 2005, SSRv, 120, 165

Burtscher, L., Davies, R. I., Gracia-Carpio, J., et al. 2015, A\&A, 586, 28

Burtscher, L., Meisenheimer, K., Tristram, K. R. W., et al. 2013, A\&A, 558, A149

Cash, W. 1979, ApJ, 228, 939

Chartas, G., Agol, E., Eracleous, M., et al. 2002, ApJ, 568, 509

Chartas, G., Kochanek, C. S., Dai, X., Poindexter, S., \& Garmire, G. 2009 ApJ, 693, 174

de Vaucouleurs, G., de Vaucouleurs, A., Corwin, H. G., Jr., et al. 1991, S\&T, $82 \mathrm{Q}, 621$

Elvis, M., Risaliti, G., Nicastro, F., et al. 2004, ApJL, 615, L25

Falco, E. E., Kurtz, M. J., Geller, M. J., et al. 1999, PASP, 111, 438

Gabriel, C., Denby, M., Fyfe, D. J., et al. 2004, in ASP Conf. Ser. 314, Astronomical Data Analysis Software and Systems (ADASS) XIII, ed. F. Ochsenbein, M. G. Allen, \& D. Egret (San Francisco, CA: ASP), 759

Gallagher, S. C., Brandt, W. N., Wills, B. J., et al. 2004, ApJ, 603, 425

Gandhi, P., Hoenig, S. F., \& Kishimoto, M. 2015, ApJ, 812, 113

Gandhi, P., Horst, H., Smette, A., et al. 2009, A\&A, 502, 457

Gandhi, P., Lansbury, G. B., Alexander, D. M., et al. 2014, ApJ, 792, 117

Gehrels, N., Chincarini, G., Giommi, P., et al. 2004, ApJ, 611, 1005

Guainazzi, M. 2002, MNRAS, 329, L13

Guainazzi, M., Matt, G., Fiore, F., \& Perola, G. C. 2002, A\&A, 388, 787

Harrison, F. A., Craig, W. W., Christensen, F. E., et al. 2013, ApJ, 770, 103

Immler, S., Brandt, W. N., Vignali, C., et al. 2003, AJ, 126, 153

Jansen, F., Lumb, D., Altieri, B., et al. 2001, A\&A, 365, L1

Kalberla, P. M. W., Burton, W. B., Hartmann, D., et al. 2005, A\&A, 440, 775

Kaspi, S., Maoz, D., Netzer, H., et al. 2005, ApJ, 629, 61

Kishimoto, M., Hönig, S. F., Antonucci, R., et al. 2011, A\&A, 527, A121

Koss, M. J., Romero-Canizales, C., Baronchelli, L., et al. 2015, ApJ, 807, 149

LaMassa, S. M., Cales, S., Moran, E. C., et al. 2015, ApJ, 800, 144

Lamer, G., Uttley, P., \& McHardy, I. M. 2003, MNRAS, 342, L41

Lansbury, G. B., Gandhi, P., Alexander, D. M., et al. 2015, ApJ, 809, 115

Longinotti, A. L., Bianchi, S., Ballo, L., de La Calle, I., \& Guainazzi, M. 2009, MNRAS, 394, L1

Madsen, K. K., Harrison, F. A., Markwardt, C., et al. 2015, ApJS, 220, 8

Magdziarz, P., \& Zdziarski, A. A. 1995, MNRAS, 273, 837

Maiolino, R., Risaliti, G., Salvati, M., et al. 2010, A\&A, 517, A47

Marchese, E., Braito, V., Della Ceca, R., Caccianiga, A., \& Severgnini, P. 2012, MNRAS, 421, 1803

Marinucci, A., Bianchi, S., Matt, G., et al. 2016, MNRAS, 456, L94

Marinucci, A., Risaliti, G., Wang, J., et al. 2013, MNRAS, 429, 2581

Markowitz, A. G., Krumpe, M., \& Nikutta, R. 2014, MNRAS, 439, 1403

Matt, G., Guainazzi, M., \& Maiolino, R. 2003, MNRAS, 342, 422

Minezaki, T., \& Matsushita, K. 2015, ApJ, 802, 98

Miniutti, G., Sanfrutos, M., Beuchert, T., et al. 2014, MNRAS, 437, 1776

Murphy, K. D., \& Yaqoob, T. 2009, MNRAS, 397, 1549

Nardini, E., \& Risaliti, G. 2011, MNRAS, 417, 2571

Peterson, B. M. 1997, An Introduction to Active Galactic Nuclei (iagn.book)

Piconcelli, E., Bianchi, S., Guainazzi, M., Fiore, F., \& Chiaberge, M. 2007, A\&A, 466, 855

Pounds, K. A., Reeves, J. N., Page, K. L., \& O’Brien, P. T. 2004, ApJ, 616, 696

Puccetti, S., Fiore, F., Risaliti, G., et al. 2007, MNRAS, 377, 607

Ricci, C., Ueda, Y., Ichikawa, K., et al. 2014a, A\&A, 567, A142 
Ricci, C., Ueda, Y., Koss, M. J., et al. 2015, ApJL, 815, L13

Ricci, C., Ueda, Y., Paltani, S., et al. 2014b, MNRAS, 441, 3622

Risaliti, G., Elvis, M., Bianchi, S., \& Matt, G. 2010, MNRAS, 406, L20

Risaliti, G., Elvis, M., Fabbiano, G., Baldi, A., \& Zezas, A. 2005, ApJL, 623, L93

Risaliti, G., Elvis, M., Fabbiano, G., et al. 2007, ApJL, 659, L111

Risaliti, G., Elvis, M., \& Nicastro, F. 2002, ApJ, 571, 234

Risaliti, G., Nardini, E., Salvati, M., et al. 2011, MNRAS, 410, 1027

Risaliti, G., Salvati, M., Elvis, M., et al. 2009, MNRAS, 393, L1

Rivers, E., Baloković, M., Arévalo, P., et al. 2015a, ApJ, 815, 55

Rivers, E., Markowitz, A., \& Rothschild, R. 2011, ApJL, 742, L29

Rivers, E., Risaliti, G., Walton, D. J., et al. 2015b, ApJ, 804, 107

Sanfrutos, M., Miniutti, G., Agís-González, B., et al. 2013, MNRAS, 436, 1588

Shappee, B. J., Prieto, J. L., Grupe, D., et al. 2014, ApJ, 788, 48
Shu, X. W., Yaqoob, T., \& Wang, J. X. 2010, ApJS, 187, 581 Stern, D. 2015, ApJ, 807, 129

Stern, D., Assef, R. J., Benford, D. J., et al. 2012, ApJ, 753, 30 Strüder, L., Briel, U., Dennerl, K., et al. 2001, A\&A, 365, L18

Suganuma, M., Yoshii, Y., Kobayashi, Y., et al. 2006, ApJ, 639, 46

Torricelli-Ciamponi, G., Pietrini, P., Risaliti, G., \& Salvati, M. 2014, MNRAS, 442, 2116

Tristram, K. R. W., \& Schartmann, M. 2011, A\&A, 531, A99

Turner, M. J. L., Abbey, A., Arnaud, M., et al. 2001, A\&A, 365, L27

Vaughan, S., Edelson, R., Warwick, R. S., \& Uttley, P. 2003, MNRAS, 345 1271

Véron-Cetty, M.-P., \& Véron, P. 2010, A\&A, 518, A10

Walton, D. J., Risaliti, G., Harrison, F. A., et al. 2014, ApJ, 788, 76

Wilms, J., Allen, A., \& McCray, R. 2000, ApJ, 542, 914

Yaqoob, T. 2012, MNRAS, 423, 3360 